\title{
Mass modelling from stellar streams in the Milky Way
}

\author{
Amina Helmi ${ }^{1}$ and Robyn E. Sanderson ${ }^{1,2,3}$ \\ ${ }^{1}$ Kapteyn Astronomical Institute, University of Groningen, P.O.Box 800, 9700 AV Groningen, \\ The Netherlands. email: ahelmi@astro.rug.nl \\ ${ }^{2}$ Columbia University Department of Astronomy, Mail Code 5246, 550 W 120th St, New York, \\ NY 10027. email: robyn@astro. columbia.edu \\ ${ }^{3} \mathrm{NSF}$ Astronomy \& Astrophysics Postdoctoral Fellow
}

\section{Introduction}

Arguably two of the most important questions in Astrophysics today are: what is the Universe made of? and, how do galaxies form and evolve? Quite astonishingly we know only the properties of $<5 \%$ of the mass in the Universe (the atoms we are made of), while the nature of the dominant mass component (the dark matter) remains elusive. However, because of its abundance, dark matter is a significant driver of both the dynamics of stars and galaxies and of their formation and evolution.

N-body simulations of the formation of structure in the Universe make very definite predictions for the distribution of mass, the shape, the time-evolution and the granularity of dark matter halos. These predictions depend strongly on the fundamental nature of the dark matter (that is, on the properties of the dark matter particles themselves). For example, if the dark matter is cold, the halos are triaxial in shape (Vera-Ciro et al. 2011), and follow specific density profiles that behave as $1 / r$ near the centres and $1 / r^{3}$ at large distances (Navarro, Frenk \& White 1996). They also host myriads of small satellites or dark matter subhalos (Springel et al. 2008). The characteristics of the dark matter halos hosting galaxies, such as their mass and scale radius, also depend slightly on the cosmological model, as systems formed in cold dark matter are more concentrated than those in warm dark matter (Lovell et al. 2014). Although many of these predictions derive from simulations that do not include the effect of baryons, these are largely only dominant in the central regions of the host halos, and therefore, such predictions should be preferably tested far out, in the outer regions of galaxies.

Tidal streams are ideally suited to test these cosmological predictions on the distribution of dark matter as well as alternative theories of gravity (Famaey \& Binney 2005). This is because stream stars follow nearly parallel orbits thereby mapping the gravitational field in which they move.

For the Milky Way, tests of the cosmological predictions are not only feasible now but can also be much more constraining because the number and variety of observations that need to be fitted simultaneously. And while much has been done, the results to-date are still inconclusive. For example the Milky Way's halo mass is not known to better than within a factor of $2(100 \%$ uncertainty). There are only weak constraints on its density profile. Its shape has received a lot of attention, yet in the past ten years its has been claimed to be round, oblate, prolate, extremely triaxial and highly unusual (Law \& Majewski 2010), or consistent with cosmological expectations if the effect of the Large Magellanic Cloud is considered (Vera-Ciro \& Helmi 2013). The reason that progress has been limited in this particular area is that very few streams (just two!) are currently known with full and reasonably accurate phase-space information (Koposov et al. 2010), 
and this information is imperative for resolving degeneracies in the modelling and for obtaining the desired tight constraints (Sanders \& Binney 2013).

However, with the launch of the Gaia mission, the situation will change dramatically in the coming years. The expectation is that Gaia will lead to the discovery of hundreds of streams (Helmi et al. 2011) that will have accurate phase-space coordinates. This of course calls for the use of sophisticated tools for the dynamical modelling.

\section{A new method to derive the mass of the Milky Way from stellar streams}

Although streams may stretch hundreds of degrees on the sky, they constitute very tight clumps in phase-space as shown in Figure 1. This is because they originate in objects that are initially compact, and this compactness is preserved in time (Liouville's theorem). The most suitable set of coordinates to describe these systems in phase-space are actionangle coordinates. These quantities are dependent on the gravitational potential, and it can be shown that the degree of clustering of streams in the space of actions is largest when these are computed for the correct gravitational potential.

Recently, Sanderson et al. (2014) have developed a statistical method based on this information content to show that the mass and scale radius of the Galactic dark halo could be recovered accurately with Gaia if assumed to be spherically symmetric. As we describe below, this is based on the application of the Kullback-Leibler (Kullback \& Leibler 1951) divergence (KLD), which can be used for clustering estimation (i.e. to determine for which values of some characteristic parameters of the potential the clustering is maximal) and also for assigning significance levels/uncertainties to the recovered parameters.

The KLD measures the relative information content of two distributions, so we use it to measure clustering in two steps. First we choose a form for the potential and trial values for its parameters a. We compute the actions $\mathbf{J}$ for a Mock sample of halo streams' stars (see below) and compare their action distribution, $f_{\mathbf{a}}(\mathbf{J})$, to the product of its marginal distributions $f_{\mathbf{a}}^{\text {shuf }}(\mathbf{J})$, constructed by shuffling each star's three actions relative to one another. Shuffling eliminates correlations between different actions, so $f^{\text {shuf }}(\mathbf{J})$ will always contain less information than $f(\mathbf{J})$. To find the best-fit potential we change the parameters of the model to maximize the KLD $\dagger$

$$
D_{\mathrm{KL}}^{\mathrm{I}}=\int f_{\mathbf{a}}(\mathbf{J}) \log \frac{f_{\mathbf{a}}(\mathbf{J})}{f_{\mathbf{a}}^{\operatorname{shuf}}(\mathbf{J})} d^{3} \mathbf{J} .
$$

Once we identify the best fit, denoted $\mathbf{a}_{0}$, we use the KLD again to compare the distribution of stellar actions computed using the best fit with the distribution for other choices of parameters $\mathbf{a}_{\text {trial }}$ (which should always be less clustered, i.e. less informative, than the distribution using $\left.\mathbf{a}_{0}\right)$ :

$$
D_{\mathrm{KL}}^{\mathrm{II}}=\int f_{\mathbf{a}_{0}}(\mathbf{J}) \log \frac{f_{\mathbf{a}_{0}}(\mathbf{J})}{f_{\mathbf{a}_{\text {trial }}}(\mathbf{J})} d^{3} \mathbf{J} .
$$

In Sanderson et al. (2014) we show that this KLD value can be interpreted as the difference in $\log$ probability of the parameters $\mathbf{a}_{0}$ and $\mathbf{a}_{\text {trial }}$, averaged over the data sample:

$$
D_{\mathrm{KL}}^{\mathrm{II}}=\left\langle\log \frac{\mathcal{P}\left(\mathcal{H}_{\mathbf{a}_{0}} \mid \mathbf{J}\right)}{\mathcal{P}\left(\mathcal{H}_{\mathbf{a}_{\text {trial }}} \mid \mathbf{J}\right)}\right\rangle_{\mathbf{J}},
$$

where $\mathcal{H}_{\mathbf{a}_{0}}$ and $\mathcal{H}_{\mathbf{a}_{\text {trial }}}$ represent the hypotheses that the distribution of $\mathbf{J}$ at the best

$\dagger$ In statistics literature this version of the KLD is known as the mutual information. 
fit came from a potential with parameters $\mathbf{a}_{0}$ or $\mathbf{a}_{\text {trial }}$, respectively. This allows us to set probability contours around the best-fit result. For instance, parameters for which $D_{\mathrm{KL}}^{\mathrm{II}}=1 / 2$ are about 24 percent as likely as the best fit (using a base- $e$ logarithm). In the case of a Gaussian distribution, this level of relative probability is attained at $1 \sigma$ on either side of the mean value, so we set this level as an analogue to a traditional $1 \sigma$ contour. Likewise $D_{\mathrm{KL}}^{\mathrm{II}}=2$ and $D_{\mathrm{KL}}^{\mathrm{II}}=9 / 2$ are roughly analogous to traditional 2- and $3 \sigma$ contours.

To test the method we construct a toy model of the stellar halo built up by 150 satellites, initially set up as Plummer spheres with mass and luminosity distributions similar to those for present-day MW satellites. We integrate the satellite's (test) particles in an isochrone potential with known parameters: the scale radius $r_{s}$ and total mass $M$. For simplicity we use one particle for each red giant star $\left(M_{V}=1\right)$ in each satellite, rather than constructing a full stellar population model. The resulting stellar streams have a uniform range of ages (integration times) so some are more phase-mixed than others. The mock halo is convolved with the Gaia pre-launch error model for end-ofmission performance and the sample for fitting is selected by (i) requiring that all six phase-space coordinates are measured by Gaia and (ii) selecting stars with estimated energy above a cutoff value to eliminate action-space crowding deep in the potential, which interferes with the fit. The fitting sample is shown in Figure 1, on the top panel in Galactic coordinates and on the bottom in a projection of action space (computed using the input potential parameters). Even when including Gaia errors the action-space distribution remains clumpy.

Using the KLD method described above, we find the best fit and produce contours in relative probability in the space of the two potential parameters, shown in the left panel of Figure 2. We pick out the contours analogous to $1(2,3) \sigma$ in green (yellow, red). Streams are most sensitive to the mass enclosed by their orbit, so we transform the $M$-axis to show the enclosed mass at the mean distance of all the stars in the fitting sample $(6.5 \mathrm{kpc})$ instead of total mass. This parameter is recovered very accurately, but there is a bias in the determination of the scale radius $r_{s}$ due to the small distance range of the stars in the fitting sample $(1-25 \mathrm{kpc})$ compared to the true scale radius $(8 \mathrm{kpc})$. If we ignore observational error momentarily and include stars for which Gaia will not measure radial velocities but ground-based spectroscopic surveys could, the upper limit on distance increases to $100 \mathrm{kpc}$ (the mean distance increases to $8.6 \mathrm{kpc}$ ) and the bias in $r_{s}$ is eliminated, as shown in the right panel of Figure 2.

\section{Conclusions}

The recently launched Gaia mission will deliver a full phase-space catalogue for more than $10^{8}$ stars in the Galaxy. It is expected that this dataset will allow us to discover several hundred streams from disrupted satellites (Helmi et al. 2011). The dynamical modeling of these streams will lead to precise estimates of the mass distribution of the dark halo of our Galaxy. Several methods have been proposed in the literature to this end, and some of the most promising include the use of clustering and correlations in actionangle space. Here we presented a new method that uses only the action information, and that can recover the mass and scale of a spherical mass distribution with $\sim 25 \%$ precision. With phase-space information from Gaia alone, the enclosed mass at the average radius is accurate to $3 \%$ but the measurement of the scale radius is biased; including radial velocities for faint stars from ground-based follow-up allows us to determine the total mass and scale radius to $10 \%$ accuracy and $20 \%$ precision. One critical advantage of the method is that it does not require the identification of the individual streams, nor the 

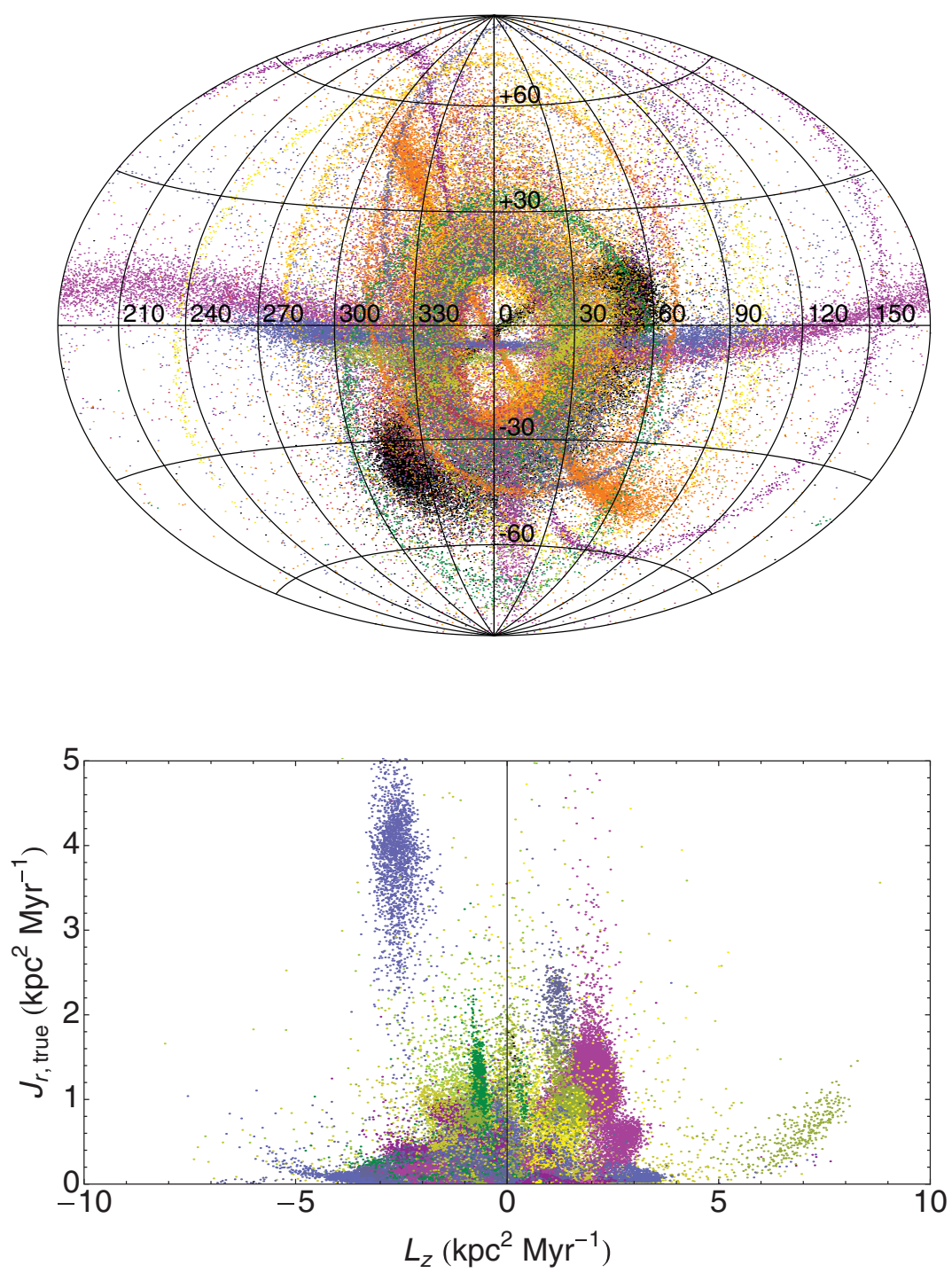

Figure 1. Tidal streams in the mock stellar halo used to test the method, in Galactic coordinates (top) and projected action space (radial action $J_{r}$ versus $z$ angular momentum $L_{z}$; bottom). Each progenitor satellite is shown in a different color.

membership of stars, and only requires that significant clustering is present in action space, as expected in the context of the concordance cosmological model (Gomez et al. 2013).

\section{Acknowledgements}

AH and RES acknowledge financial support from European Research Council under ERCStG grant GALACTICA-240271. RES is supported by the National Science Foundation under Award No. AST-1400989. 

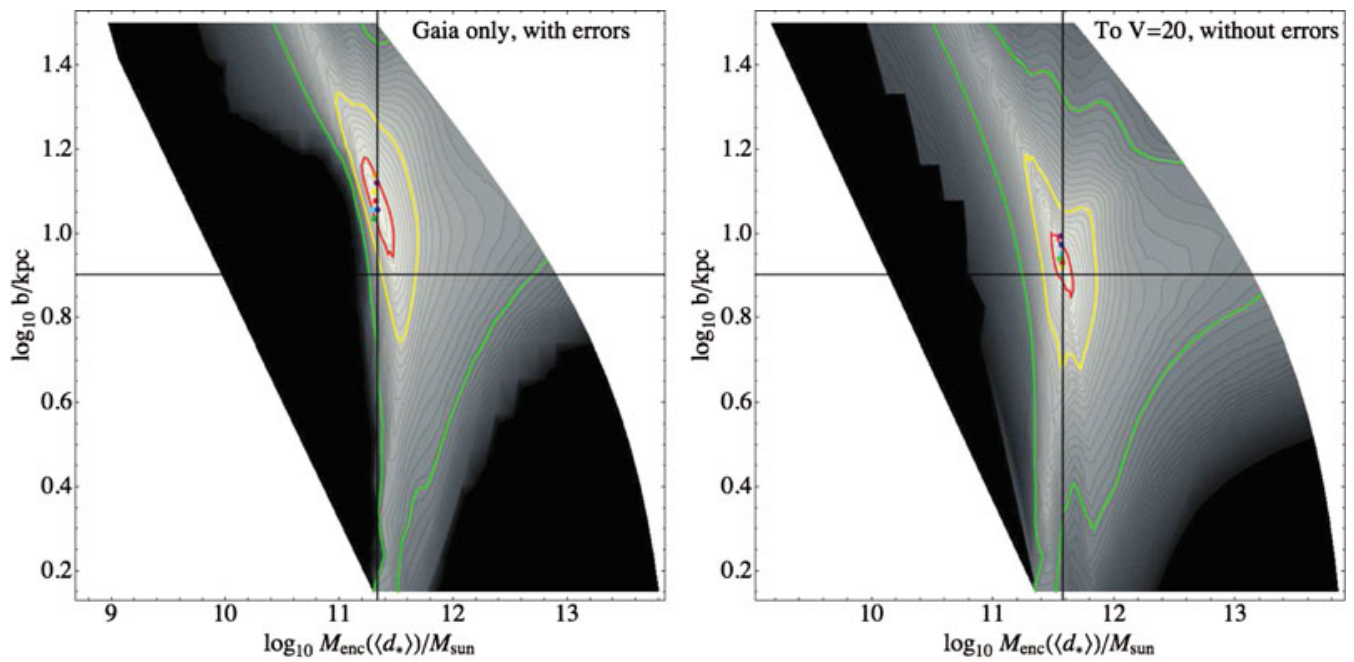

Figure 2. Contours of $D_{\mathrm{KL}}^{\mathrm{II}}$ in scale radius $b$ and enclosed mass $M_{\mathrm{enc}}$ at the average distance of stars in the sample $\left\langle d_{*}\right\rangle$, for two different fitting samples. On the left are results from the error-convolved fitting sample when including only stars with Gaia radial velocities. On the right are results from a sample without errors that includes all stars for which Gaia will measure proper motions, including those whose radial velocities must be obtained by follow-up from the ground. In both panels the red (yellow, green) contours show the $D_{\mathrm{KL}}^{\mathrm{II}}=1 / 2(2,9 / 2)$ levels, analogous to $1(2,3) \sigma$ in the Gaussian case; the thin contours show steps of 0.2 in $D_{\mathrm{KL}}^{\mathrm{II}}$. The solid crosshairs intersect at the input values of the parameters; the colored points (red through purple) are the first through seventh highest values of $D_{\mathrm{KL}}^{\mathrm{I}}$, which is maximized to find the best-fit parameters (red).

\section{References}

Famaey, B. \& Binney, J., 2005, MNRAS, 363, 603

Gomez, F. A., Helmi, A., et al. 2013, MNRAS 436, 3602

Helmi et al., 2011, ApJ, 733, L7

Koposov, S. E., Rix, H.-W., \& Hogg, D. W. 2010, ApJ, 712, 260

Kullback, S. \& Leibler, R. A. 1951, The Annals of Mathematical Statistics, 22, 79

Law, D. R. \& Majewski, S. R. 2010, ApJ, 714, 229

Lovell, M. R., Frenk, C. S., Eke, V. R., et al. 2014, MNRAS, 439, 300

Navarro J., Frenk C. \& White S. 1996, ApJ, 462, 563

Sanders, J. L. \& Binney, J. 2013, MNRAS, 433, 1826

Sanderson, R., Helmi, A., \& Hogg, D. W. 2014, Action-space clustering of tidal streams to infer the Galactic potential, submitted to ApJ, arXiv:1404.6534

Springel V. et al. 2008, MNRAS, 391, 1685

Vera-Ciro, C. \& Helmi, A. 2013, ApJ, 773, L4

Vera-Ciro, C. A., Sales, L. V., Helmi, A., et al. 2011, MNRAS, 416, 1377 